\title{
High-Flow Nasal Cannula Therapy With Early Extubation for Subjects Undergoing Off-Pump Coronary Artery Bypass Graft Surgery
}

\author{
Wataru Tatsuishi, Toshiro Sato, Go Kataoka, Atsuhiko Sato, Ryota Asano, and Kiyoharu Nakano
}

\begin{abstract}
BACKGROUND: The effects of high-flow nasal cannula (HFNC) therapy on postoperative atelectasis and duration of oxygen therapy after off-pump coronary artery bypass graft are unknown. The purpose of this study was to compare the effects of HFNC therapy for subjects who underwent off-pump coronary artery bypass graft with the effects of standard oxygen therapy in terms of oxygen requirement and atelectasis. METHODS: This prospective single-blinded randomized, controlled trial included 148 subjects who underwent off-pump coronary artery bypass graft between 2010 and 2015 with HFNC $(n=72)$ or without HFNC (standard $\left.O_{2}, n=76\right)$. The primary end point was the percentage difference in loss of lung volume between subjects with or without HFNC therapy. Secondary end points included the total amount of oxygen administered and duration of oxygen therapy with and without HFNC therapy. RESULTS: There were significant between-group differences in the percentage loss of lung volume $(P<.001)$, total amount of oxygen administered $(P<.001)$, duration of oxygen therapy $(P<.001)$, and the need for postoperative diuretic therapy $(P=.037)$. The amount $(\rho=0.569, P<.001)$ and duration $(\rho=0.678, P<.001)$ of oxygen administered were correlated with atelectasis volume. CONCLUSIONS: Using HFNC therapy after off-pump coronary artery bypass graft shortened the duration of oxygen therapy and reduced the percentage loss of lung volume and total amount of oxygen administered when compared with standard oxygen therapy. Key words: high-flow nasal cannula therapy; coronary artery bypass grafting; atelectasis; respiration. [Respir Care 2020;65(2):183-190. (C) 2020 Daedalus Enterprises]
\end{abstract}

\section{Introduction}

Effective respiratory management is one of the most important priorities after cardiac surgery. Postoperative atelectasis has been associated with the duration of oxygen therapy and delay in resumption of activities of daily living after surgery. ${ }^{1}$ Furthermore, atelectasis compromises

Drs Tatsuishi, Kataoka, Sato, Asano, and Nakano are affiliated with the Department of Cardiovascular Surgery, and Mr Sato is affiliated with the Department of Clinical Engineering, Tokyo Women's Medical University, Medical Center East, Tokyo, Japan.

They authors have declared no conflicts of interest.

Correspondence: Wataru Tatsuishi MD PhD, Department of Cardiovascular Surgery, Tokyo Women's Medical University, Medical Center East, Nishiogu 2-1-10, Arakawa-Ku, Tokyo 116-8567, Japan. E-mail: wataru0812_drt@yahoo.co.jp.

DOI: $10.4187 /$ respcare.06382 successful recovery after surgery and may prolong the hospital stay. ${ }^{1}$ At our institution, all coronary artery bypass graft surgery is performed off-pump in the belief that offpump coronary artery bypass graft surgery is less invasive than the same procedure on-pump, and that respiration after off-pump coronary artery bypass graft surgery would be better than that after the same procedure on-pump. However, the loss of lung volume seen on postoperative chest films and computed tomography scans was larger than anticipated.

In recent years, HFNC therapy has been introduced in postoperative respiratory care, and its efficacy has been well described. ${ }^{2-4}$ HFNC therapy delivers a high flow of humidified gas at a level near the patient's required inspiratory flow ${ }^{2-4,7,8}$ while maintaining a constant oxygen concentration and humidified inhalation via a specialized nasal interface. HFNC therapy consists of an oxygen/air blender, a heated humidifier, a single heated circuit, and a nasal cannula. Flow can be titrated up to $60 \mathrm{~L} / \mathrm{min}$, and the oxygen concentration can be titrated between $21 \%$ and 
$100 \%$. PEEP generated with HFNC therapy has been reported to achieve an increase in functional residual volume, reduces air trapping, and a reduction in inspiratory threshold load. ${ }^{3,5-7}$ Therefore, HFNC therapy improves oxygenation, reduces expiratory and inspiratory work, and improves alveolar ventilation. Although these effects are also achieved with the use of a CPAP mask, HFNC is more comfortable than CPAP. ${ }^{9}$ Moreover, HFNC may wash out intraoral expiratory carbon dioxide and creates a reservoir of oxygen in the nasopharyngeal cavity, because the high-flow supply can flush and replace the dead space volume. ${ }^{8,10}$

Washout of nasopharyngeal dead space reduces the rate of re-intubation. Hernández et $\mathrm{al}^{11}$ reported that HFNC therapy was noninferior to noninvasive ventilation with regard to avoidance of the need for postoperative re-intubation. Accordingly, we hypothesized that HFNC therapy could reduce the loss of lung volume and shorten the required duration of oxygen therapy, and we introduced HFNC therapy for postoperative support of subjects undergoing off-pump coronary artery bypass graft surgery.

The aim of this study was to investigate the efficacy of HFNC therapy in subjects undergoing coronary artery bypass graft surgery in terms of postoperative atelectasis and duration of oxygen therapy.

\section{Methods}

\section{Study Groups}

A total of 158 consecutive patients who underwent offpump coronary artery bypass graft surgery at Tokyo Women's Medical University from January 2010 to May 2015 were selected to participate in this prospective singleblinded randomized controlled trial. None of the subjects refused to be enrolled, and all provided written informed consent for cardiac multidetector-row computed tomography (MDCT) and coronary artery bypass graft surgery. The study protocol was approved by the institutional review board at Tokyo Women's Medical University (approval number 4397). Patients undergoing concomitant procedures, such as valve surgery, aortic surgery, and congenital cardiac surgery, were not in the selected group because of the need for cardiopulmonary bypass. Patients with chronic kidney disease (serum creatinine $\geq 2.0 \mathrm{mg} / \mathrm{dL}$ without dialysis) were excluded because of concerns regarding renal injury due to the contrast medium used with postoperative cardiac MDCT $(n=7)$. Patients who withdrew from HFNC therapy because of discomfort were also excluded $(n=3)$. Finally, 148 subjects were enrolled, 113 of whom were men. Preoperatively, subjects were randomly allocated receive HFNC therapy $(n=72)$ or a standard $\mathrm{O}_{2}$ mask $(n=76)$ after coronary artery bypass graft surgery. The method of randomization was by coin

\section{QUICK LOOK}

\section{Current knowledge}

The effects of postoperative HFNC therapy have been well described. However, previous reports have focused on the effect that HFNC therapy has on PEEP and avoidance of re-intubation.

\section{What this paper contributes to our knowledge}

HFNC therapy with early extubation in subjects who had undergone off-pump coronary artery bypass graft improved postoperative atelectasis and shortened the duration of oxygen therapy when compared with standard oxygen therapy. A positive effect of HFNC therapy on postoperative atelectasis was confirmed with computed tomography images.

toss (tails meaning allocation to HFNC therapy). A flow chart outlining the steps of this study is shown in Figure 1.

\section{Surgical Procedure}

A full median sternotomy and off-pump coronary artery bypass graft surgery were performed in all subjects. The goal of coronary artery bypass graft surgery is to achieve complete functional revascularization. Left and right internal thoracic artery grafts were grafted exclusively to the left anterior descending artery or the left circumflex artery; saphenous vein grafts and the right gastroepiploic artery were grafted to the right coronary artery.

\section{Postoperative Management}

Circulation Management. Fluid management was achieved using goal-directed fluid management. ${ }^{12}$ Hemodynamic monitoring was performed using a Swan-Ganz catheter. The circulation indices were mixed venous oxygen saturation, cardiac index, systolic volume index, and the end-diastolic volume index. Dopamine, nitroglycerin, and phosphodiesterase inhibitors, in addition to intravenous volume expanders, were often administered intraoperatively and postoperatively to stabilize the hemodynamic parameters. The catecholamine volume administered was reduced by postoperative day 1 ; volume administration was discontinued on postoperative day 1 in almost all subjects $(n=141)$ without antibiotics. Oral fluids were restricted postoperatively $(1,000 \mathrm{~mL} / \mathrm{d})$ to avoid accumulation of pleural and pericardial effusion. A diuretic was administered $5 \mathrm{~d}$ after surgery if postoperative drainage was poor (ie, $>2 \mathrm{~kg}$ compared with preoperatively) or when pleural effusion did not decrease. 


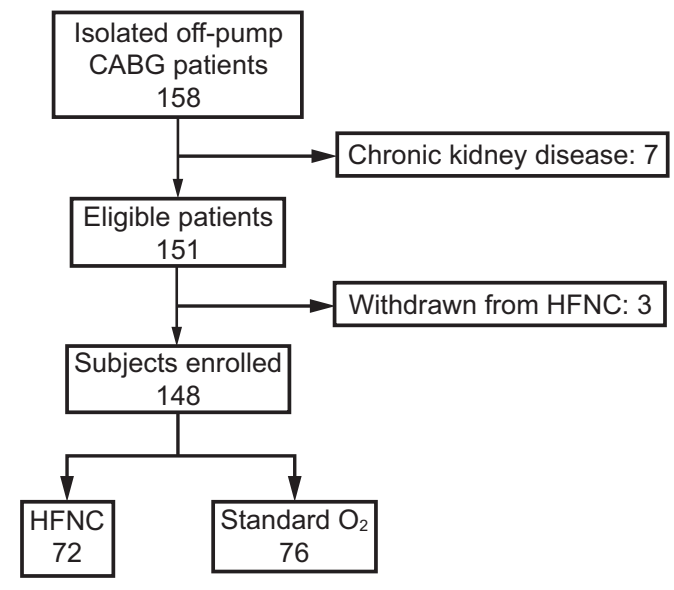

Fig. 1. Flow chart. HFNC = high-flow nasal cannula, $\mathrm{CABG}=$ coronary artery bypass graft.

Ventilator Management. Following coronary artery bypass graft surgery, all subjects were transferred to the ICU while still intubated. A ventilator (Evita XL or Evita V500, Dräger Medical, Lübeck, Germany) was used to provide postoperative mechanical ventilation. A recruitment maneuver (3-breath, high PEEP; 55$70 \mathrm{~cm} \mathrm{H}_{2} \mathrm{O}, 2-3 \mathrm{~s}, 3$ times) was performed to open alveoli and to achieve $\mathrm{P}_{\mathrm{aO}_{2}} / \mathrm{F}_{\mathrm{IO}_{2}}>500 \mathrm{~mm} \mathrm{Hg}$. Furthermore, a low-flow pressure-volume closed loop was used to establish the optimal level of PEEP required to keep alveoli open; thereafter, biphasic intermittent positive airway pressure management was used. The mean PEEP was $13 \pm 3 \mathrm{~mm} \mathrm{Hg}$. After spontaneous breathing was confirmed, weaning was performed using the Smart Care function, which automatically determines the appropriate level of ventilator support. ${ }^{13}$ This ventilation management strategy was used in both groups.

The extubation criteria were satisfactory respiratory status (with 5-8 $\mathrm{cm} \mathrm{H}_{2} \mathrm{O}$ PEEP and 21-40\% $\mathrm{F}_{\mathrm{IO}_{2}}$ ), no bleeding, and a normal level of consciousness. After extubation, HFNC therapy with $45-60 \mathrm{~L} / \mathrm{min}$ and $21-35 \% \mathrm{~F}_{\mathrm{IO}_{2}}$ were used in the HFNC group; a flow of 3-12 L/min and $100 \%$ oxygen via a standard oxygen mask with humidification were used in the standard $\mathrm{O}_{2}$ group. The amount of oxygen was set to maintain normal mixed venous oxygen saturation $\left(\mathrm{S}_{\overline{\mathrm{v}}} \mathrm{O}_{2} ; 60-80 \%\right)$ or to avoid a low $\mathrm{S}_{\mathrm{pO}_{2}}(<90 \%)$ on the day of surgery and on postoperative day 1 in the ICU. The total oxygen volume was calculated as follows: flow of oxygen/min $\times$ administration minutes by every level of oxygen concentration.

Rehabilitation Management. Almost all subjects $(n=$ 142) were returned to the ward from the ICU on postoperative day 1 . Walking rehabilitation was implemented by nurses or physiotherapists. The target $\mathrm{S}_{\mathrm{pO}_{2}}$ after return to the hospital ward was approximately $\geq 94 \%\left(\mathrm{~S}_{\mathrm{pO}_{2}} \geq 90 \%\right.$ in subjects with COPD) with no additional symptoms such as shortness of breath in either group, and support was maintained using a standard face mask or nasal cannula. When the target $\mathrm{S}_{\mathrm{pO}_{2}}$ was maintained, the amount of oxygen was gradually reduced by $1 \mathrm{~L} / \mathrm{min}$.

\section{Image Reconstruction and Volume Calculation}

Postoperative graft patency is evaluated at our institution using cardiac electrocardiographically gated 64-column MDCT on postoperative day 5. All cardiac MDCT examinations were performed using a LightSpeed VCT XT scanner (GE Healthcare, Waukesha, Wisconsin). In this study, cardiac MDCT was used to evaluate the lung volume loss in coronary artery bypass graft surgery. All reconstructed images were reviewed in axial projection; subsequently, image tools (Zio Station, Tokyo, Japan) were used to examine the images. After 3-dimensional image reconstruction using a volume-rendering procedure, the volume on both sides of the chest cavity and the lung volume loss and pleural effusion were measured using the differences in the computed tomography values (ie, the Zio station can automatically measure these volumes; total lung volume was defined as both sides of the chest cavity). The amount of lung volume loss was defined as the total of atelectasis and pleural effusion volumes. The percentage of the loss of lung volume against the chest cavity was calculated and compared with and without HFNC therapy, which is the primary end point (Fig. 2).

We also examined electrical impedance tomography ventilation images (Dräger PulmoVista500, Dräger Medical United Kingdom, Hemel Hempstead, United Kingdom) for each oxygen concentration. Electrical impedance tomography is a technique used to acquire real-time images and data from regional lung ventilation and lung volumes at the bedside. ${ }^{14} \mathrm{We}$ compared the data obtained at 5 $\mathrm{L} / \mathrm{min}$ with a standard $\mathrm{O}_{2}$ mask and at $30 \mathrm{~L} / \mathrm{min}, 40 \mathrm{~L} / \mathrm{min}$, and $50 \mathrm{~L} / \mathrm{min}$ with HFNC therapy.

\section{Statistical Analysis}

The primary end point of this study was a reduction in the percentage of lung volume loss. The secondary end points were improvement in the total amount of oxygen administered from the time of extubation and the duration of oxygen inhalation therapy from the time of extubation. Correlations between the loss of lung volume and various factors were examined. Medical staff involved were blinded to the data and the results. The sample size was calculated by the effect size when comparing the loss of lung volume between the study groups. We assumed a 5\% difference in the mean and a $7.5 \%$ difference in the standard deviation. As a result, the effect size was 0.67 , and the minimum required sample size was 49 cases in each group when 


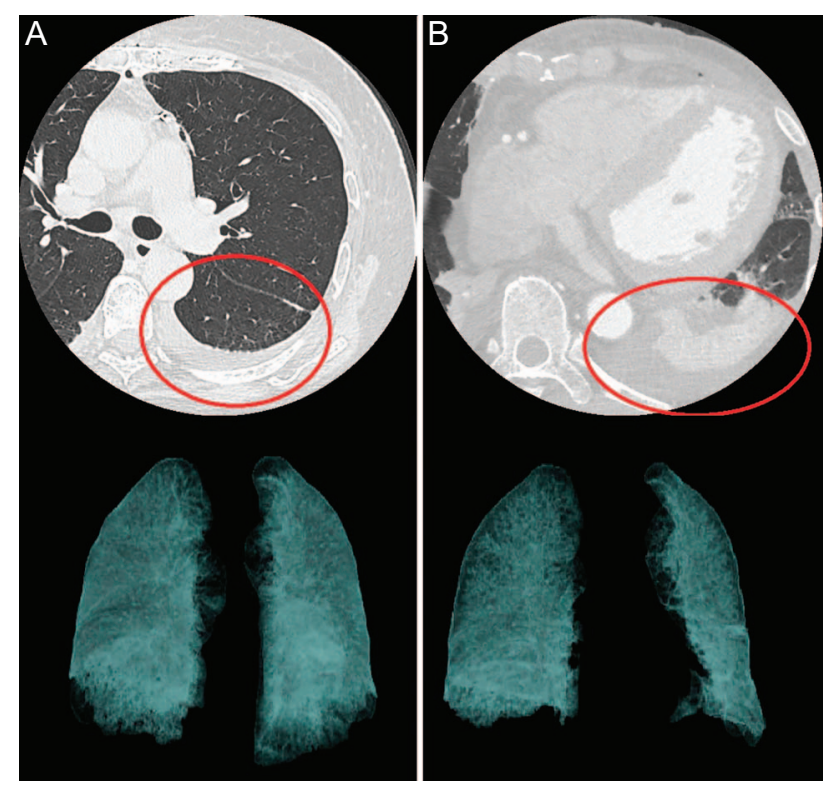

Fig. 2. Comparison of postoperative multidetector-row computed tomography and 3-dimensional volume evaluation of atelectasis after coronary artery bypass surgery between A: HFNC and B: standard $\mathrm{O}_{2}$, reconstructed with image tools. These images shows the effectiveness of HFNC. Red circles indicate atelectasis and pleural effusion, and volume was larger with standard $\mathrm{O}_{2}$ than with HFNC; 3-dimensional expanded lung volume was larger with HFNC than with standard $\mathrm{O}_{2}$. HFNC = high-flow nasal cannula.

calculated with a power of $95 \%$ and a significance level of $5 \%$. We targeted approximately 160 cases for the total number of samples, considering the number of subjects who would be excluded.

JMP Pro 11 (SAS Institute, Cary, North Carolina) was used to analyze the data. Normality was tested with the Shapiro-Wilk test. To compare the characteristics of the 2 study groups, the following tests were performed: the independent $t$ test (for continuous variables with a normal distribution); the Mann-Whitney $U$ test (for continuous variables with a non-normal distribution); and the chisquared test. $P<.05$ was considered statistically significant. Correlations were examined using the Spearman's rank correlation coefficient. The data are presented as the mean \pm standard deviation or as the median (interquartile range $[\mathrm{IQR}])$ in the text and tables.

\section{Results}

The baseline characteristics of the subjects are summarized in Tables 1 and 2. The duration of HFNC therapy was $18 \pm 6 \mathrm{~h}$. The postoperative drainage volume was significantly larger in the HFNC group than in the standard $\mathrm{O}_{2}$ group $(P<.001)$; however, no subject underwent any further surgical intervention for bleeding. Otherwise, there were no significant differences between the two groups at baseline.
Table 1. Clinical Characteristics of Subjects

\begin{tabular}{lccc}
\hline \hline \multicolumn{1}{c}{ Variable } & HFNC & Standard $\mathrm{O}_{2}$ & $P$ \\
\hline Male & $57(79.2)$ & $56(73.7)$ & $.43 \dagger$ \\
Age, y & $69 \pm 7$ & $69 \pm 10$ & $.33 \dagger$ \\
Body surface area, $\mathrm{m}^{2}$ & $1.66 \pm 0.15$ & $1.65 \pm 0.19$ & $.42 \dagger$ \\
Hypertension & $61(84.7)$ & $65(85.5)$ & $.89 \dagger$ \\
Dyslipidemia & $49(68.1)$ & $58(76.3)$ & $.26 \dagger$ \\
Renal insufficiency* & $31(44.3)$ & $25(33.3)$ & $.17 \dagger$ \\
Dialysis & $10(13.9)$ & $7(9.2)$ & $.37 \dagger$ \\
Diabetes mellitus & $40(55.6)$ & $44(57.9)$ & $.77 \dagger$ \\
Old myocardial infarction & $28(38.9)$ & $31(41.3)$ & $.76 \dagger$ \\
Past percutaneous coronary & $15(20.1)$ & $17(22.4)$ & $.82 \dagger$ \\
$\quad$ intervention & & & \\
Cerebral infarction & $10(13.9)$ & $10(13.2)$ & $.90 \dagger$ \\
Peripheral vascular disease & $8(11.1)$ & $6(7.9)$ & $.50 \dagger$ \\
Smoking history & $42(58.3)$ & $47(61.8)$ & $.66 \dagger$ \\
COPD & $3(4.2)$ & $3(3.9)$ & $.71 \dagger$ \\
Low ejection fraction & $5(6.9)$ & $3(4.0)$ & $.42 \dagger$ \\
$\quad$ (ie, $<30 \%)$ & & &
\end{tabular}

Data are presented as $n(\%)$ or mean \pm SD. HFNC: $n=72$ subjects; Standard $\mathrm{O}_{2}$ : $n=76$ subjects.

* Renal insufficiency defined as estimated glomerular filtration rate $<60 \mathrm{~mL} / \mathrm{min} / 1.73 \mathrm{~m}^{2}$ $\dagger$ Chi-square test.

‡ Independent $t$ test.

HFNC $=$ high-flow nasal cannula

There were significant between-group differences in the total volume of oxygen administered from the time of extubation $(4,650 \mathrm{~L}$ [IQR 3,240-7,140] in HFNC and 11,760 L [IQR 9,720-15,090] in standard $\mathrm{O}_{2}, P<.001$ ) and in the duration of oxygen inhalation therapy from the time of extubation (30 h [IQR 18-40] in HFNC and $57 \mathrm{~h}$ [IQR 40-68] in standard $\mathrm{O}_{2}, P<.001$; Fig. 3). Thirtyfive HFNC subjects and 11 standard $\mathrm{O}_{2}$ subjects did not require oxygen inhalation therapy on postoperative day 1 $(P<.001)$, and 28 and 37, respectively, did not require it on postoperative day $2(P<.001)$. The percentage of loss of lung volume observed in the chest cavity was significantly smaller in the HFNC group than in the standard $\mathrm{O}_{2}$ group $(5.2 \pm 2.2 \%$ vs $9.2 \pm 2.2 \%$, respectively, $P<.001)$. Furthermore, the need for postoperative diuretic therapy because of prolonged retention of fluid and pleural fluid was significantly lower in subjects undergoing HFNC $(P=.037$, Table 2).

The Spearman rank correlation coefficient test indicated a moderately strong correlation between duration of oxygen inhalation therapy and loss of lung volume ( $\rho=0.569$, $P<.001)$. In addition, the total amount of oxygen administered was strongly related to loss of lung volume ( $\rho=0.678, P<.001$; Fig. 4$)$. There was a weak correlation between intubation time and loss of lung volume $(\rho=0.342, P<.001)$. Although the stay in the ICU was the same for both groups, the overall hospital stay was shorter for HFNC subjects than for standard $\mathrm{O}_{2}$ subjects 
Table 2. Operative Characteristics of Subjects

\begin{tabular}{|c|c|c|c|}
\hline Variable & HFNC & Standard $\mathrm{O}_{2}$ & $P$ \\
\hline Clinical status & & & $.34 *$ \\
\hline Elective & $61(84.7)$ & $66(86.8)$ & \\
\hline Urgent & $6(8.3)$ & $5(6.6)$ & \\
\hline Emergent & $5(6.9)$ & $5(6.6)$ & \\
\hline Congestive heart failure & $21(19.8)$ & $26(22.8)$ & $.64 *$ \\
\hline Left main trunk disease & $12(16.7)$ & $22(29.0)$ & $.08^{*}$ \\
\hline Re-do operation & $2(2.8)$ & $1(1.3)$ & $.57 *$ \\
\hline Intra-aortic balloon pumping & $10(13.9)$ & $6(7.9)$ & $.22 *$ \\
\hline Number of coronary stenosis & $2.5 \pm 0.1$ & $2.4 \pm 0.1$ & $.20 \dagger$ \\
\hline Anastomosis & $3.0(2.0-3.0)$ & $3.0(2.0-3.0)$ & $.64 \%$ \\
\hline Bilateral internal thoracic artery & $40(52.0)$ & $37(48.7)$ & $.40^{*}$ \\
\hline Operative time, $\min$ & $173 \pm 43$ & $182 \pm 52$ & $.22 \dagger$ \\
\hline Intraoperative hemorrhage, $\mathrm{mL}$ & $500(340-725)$ & $500(300-687.5)$ & $.86 \neq$ \\
\hline Blood transfusion & $8(7.5)$ & $11(9.6)$ & $.58^{*}$ \\
\hline $\begin{array}{l}\text { Postoperative drainage volume, } \\
\mathrm{mL}\end{array}$ & $410(285-523.8)$ & $318(221.3-420)$ & $<.001$ \\
\hline Ventilation duration, $\mathrm{h}$ & $2(1.5-2.5)$ & $2.5(1.85-3)$ & $.10 \ddagger$ \\
\hline Volume balance, $\mathrm{mL}$ & $3,380 \pm 521$ & $3,250 \pm 473$ & $.27 \dagger$ \\
\hline ICU LOS, d & $1(1-1)$ & $1(1-1)$ & $.65 \ddagger$ \\
\hline Postoperative hospital LOS, d & $8(7-10)$ & $9(7-12)$ & .056 \\
\hline $\begin{array}{l}\text { Postoperative diuretic } \\
\text { administration }\end{array}$ & $5(6.9)$ & $14(18.4)$ & $.037^{*}$ \\
\hline $\begin{array}{l}\text { Data are presented as } n(\%), n \text { (interq } \\
\text { Standard } \mathrm{O}_{2}: n=76 \text { subjects. } \\
* \text { Chi-square test. } \\
\dagger \text { Independent } t \text { test. } \\
\ddagger \text { Mann-Whitney } U \text { test. } \\
\text { HFNC = high-flow nasal cannula } \\
\text { LOS = length of stay }\end{array}$ & artile range), or mean & \pm SD. HFNC: $n=72$ & subjects; \\
\hline
\end{tabular}

(8 d [IQR 7-10] vs $9 \mathrm{~d}$ [IQR 7-12]), but the difference was not statistically significant $(P=.056$; Table 2$)$.

Figure 5 shows the electrical impedance tomography study, which revealed that the larger the flow, the better the expansion of the lungs; and sufficient expansion of the alveoli was achieved by only $50 \mathrm{~L} / \mathrm{min}$. No adverse events related to the use of HFNC therapy were recorded.

\section{Discussion}

In this study, use of HFNC therapy was associated with a reduction in both postoperative atelectasis and duration of oxygen therapy. Although various reports in the past have illustrated the effect of HFNC in cardiac surgery, ${ }^{14,15}$ this report demonstrates the effect of HFNC therapy using images of postoperative atelectasis volume. Furthermore, HFNC therapy might also aid early postoperative rehabilitation.

The duration of oxygen therapy was short in the HFNC group $(P<.001)$. The authors attribute this result to the postoperative respiratory measures used (such as the recruitment maneuver and the ability of PEEP to maintain alveolar recruitment) in combination with HFNC therapy. Landquist et al ${ }^{16}$ reported that atelectasis occurred in $87 \%$ of subjects who had undergone anesthesia or mechanical ventilation. Atelectasis has been observed in up to $90 \%$ of subjects who undergo cardiac surgery, ${ }^{17}$ particularly in the dependent lung (ie, the dorsal side). The aim during this study was to achieve effective alveolar ventilation by avoiding collapse of the alveoli. Futier et al ${ }^{18}$ reported that use of HFNC therapy after extubation did not improve pulmonary outcomes in subjects who had undergone major abdominal surgery; however, the PEEP used in their study was relatively lower $(6.2 \pm 1.3 \mathrm{~mm} \mathrm{Hg})$ than that used in our study and may have resulted in insufficient recruitment of the dependent lung regions. Outcome measures demonstrated that many subjects in the HFNC group had less atelectasis in the dependent lung on postoperative day 1. The difference in flow effect using HFNC therapy was important. The electrical impedance tomography study suggested that dorsal lung ventilation was better on $50 \mathrm{~L} / \mathrm{min}$ than on $<40 \mathrm{~L} / \mathrm{min}$ (Fig. 5). It also suggested a positive relationship between PEEP and flow quantity, and its counterbalance of auto-PEEP and reduced work of breathing. ${ }^{5,19}$ Furthermore, Zochios et al ${ }^{15}$ reported that a PEEP of 4.0 $7.1 \mathrm{~cm} \mathrm{H}_{2} \mathrm{O}$ was observed at $60 \mathrm{~L} / \mathrm{min} \mathrm{HFNC}$, and Parke et $\mathrm{al}^{5}$ observed $1.93 \pm 1.25 \mathrm{~cm} \mathrm{H}_{2} \mathrm{O}, 2.58 \pm 1.54 \mathrm{~cm} \mathrm{H}_{2} \mathrm{O}$, and $3.31 \pm 1.05 \mathrm{~cm} \mathrm{H}_{2} \mathrm{O}$ at 30,40 , and $50 \mathrm{~L} / \mathrm{min}$ HFNC with the mouth closed. The pressures reported by Ritchie et $\mathrm{al}^{6}$ are similar to those reported by Parke et $\mathrm{al}^{5}$ $\left(1.5 \pm 0.7 \mathrm{~cm} \mathrm{H}_{2} \mathrm{O}, 2.2 \pm 0.8 \mathrm{~cm} \mathrm{H}_{2} \mathrm{O}\right.$, and $3.1 \pm 1.2 \mathrm{~cm} \mathrm{H}_{2} \mathrm{O}$ at 40,50 , and $60 \mathrm{~L} / \mathrm{min}$ HFNC, respectively). From these reports and our present findings, we suggest that HFNC therapy not only results in low PEEP but also has an effect on flow delivery. This effect is greater as the flow increases and could further improve the flow of oxygen to both the dependent and non-dependent lungs.

Shortening the duration of oxygen inhalation typically reduced the total amount of oxygen that the subjects received. Moreover, administration of high-concentration oxygen was not needed because of the effects of HFNC therapy, which included improved oxygenation, a reduction in work of breathing, and improved functioning of alveolar ventilation. Reduced $\mathrm{F}_{\mathrm{IO}_{2}}$ levels are often required with HFNC systems compared to the air-entrainment mask, secondary to differences in system delivery. A standard $\mathrm{O}_{2}$ mask or nasal cannula cannot supply a flow greater than a patient's inspiratory volume; therefore, inspiratory flow was a mixture of the oxygen supplied and entrained air. As a result, when using a standard mask or nasal cannula, the fraction of delivered oxygen was less than the $\mathrm{F}_{\mathrm{IO}_{2}}$. Conversely, with HFNC therapy, the flow supplied matched the subject's inspiratory volume; therefore, the deviation between the fraction of delivered oxygen and $\mathrm{F}_{\mathrm{IO}_{2}}$ was extremely small and well titrated. In this study, the amount of oxygen administered in the HFNC group was approximately $60 \%$ of the amount of oxygen in the standard $\mathrm{O}_{2}$ group during their ICU stay. There was a correlation between the duration of oxygen therapy and atelectasis volume, and the total amount of oxygen administered was 

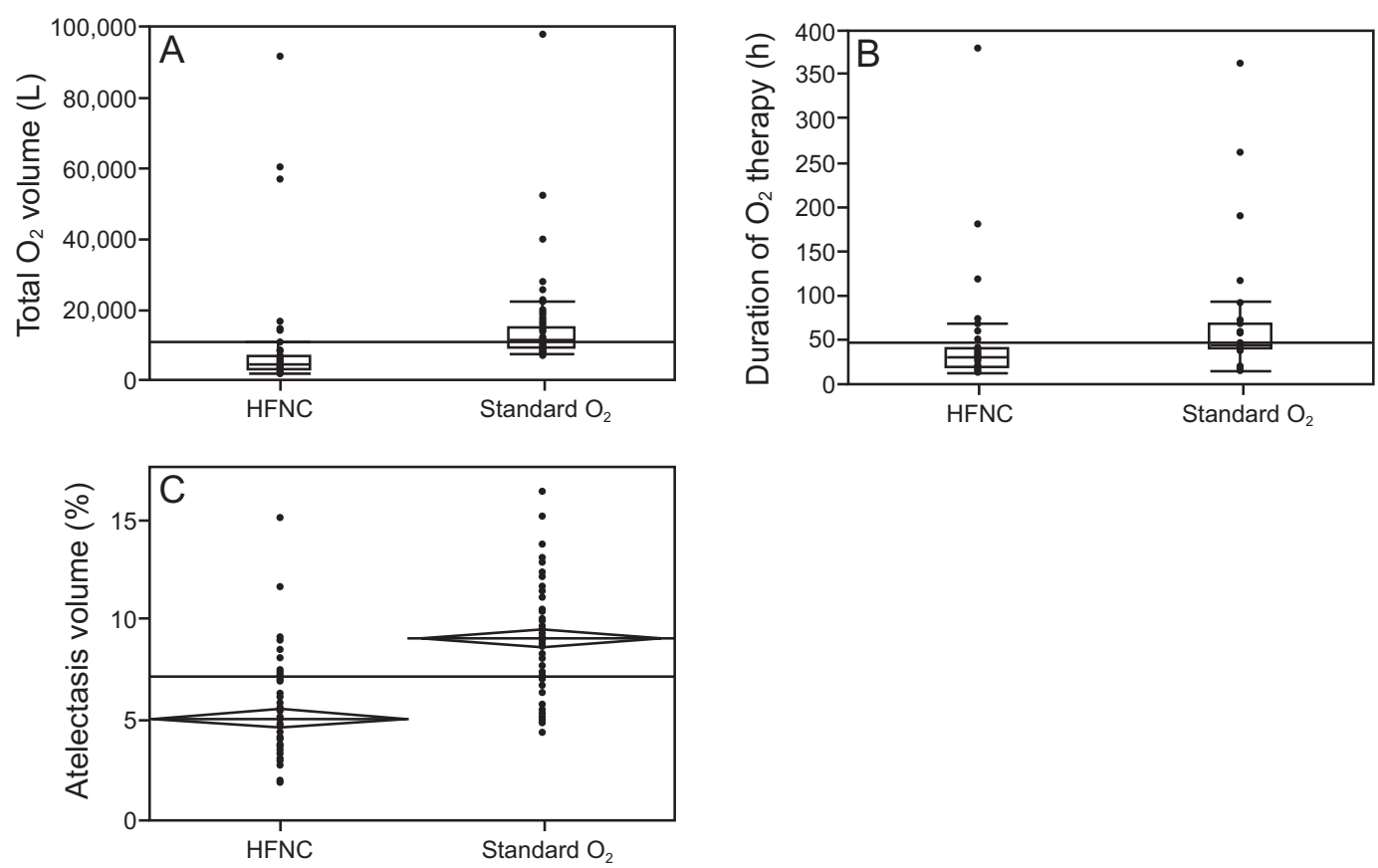

Fig. 3. A: The total volume of $\mathrm{O}_{2}$ for subjects from the time of extubation. B: The duration of $\mathrm{O}_{2}$ therapy from the time of extubation. C: Comparison of lung volume loss between $\mathrm{HFNC}$ and standard $\mathrm{O}_{2}$.
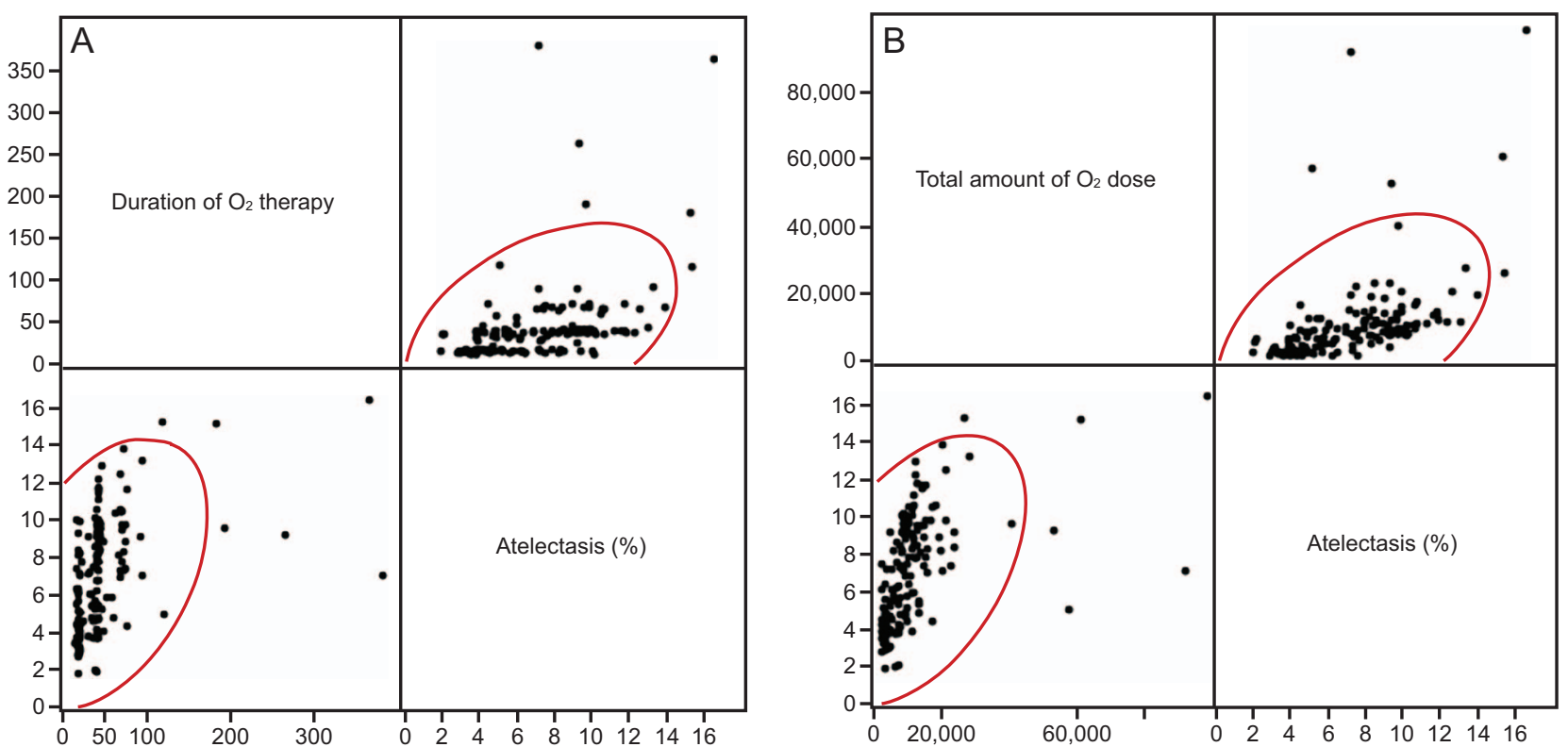

Fig. 4. The results of Spearman's rank correlation coefficient testing: (A) between duration of $\mathrm{O}_{2}$ therapy and percentage of atelectasis volume $(\rho=0.678 ; P<.001)$ and $(B)$ between total amount of $\mathrm{O}_{2}$ dose and percentage of atelectasis volume $(\rho=0.569, P<.001)$.

also related to the atelectasis volume. These results mean that HFNC therapy has a direct effect. Using HFNC therapy reduced the atelectasis volume, and, as a result, the subject's oxygen requirement was reduced. Therefore, the duration of oxygen therapy and the amount of oxygen administered were decreased.
Our institution has implemented the ERAS (Enhanced Recovery After Surgery) protocol for perioperative management, ${ }^{20}$ and other institutions have reported fast-track management for coronary artery bypass graft. ${ }^{21,22}$ One concept in this regimen is early postoperative rehabilitation. In this study, almost all subjects were transferred to the ward 

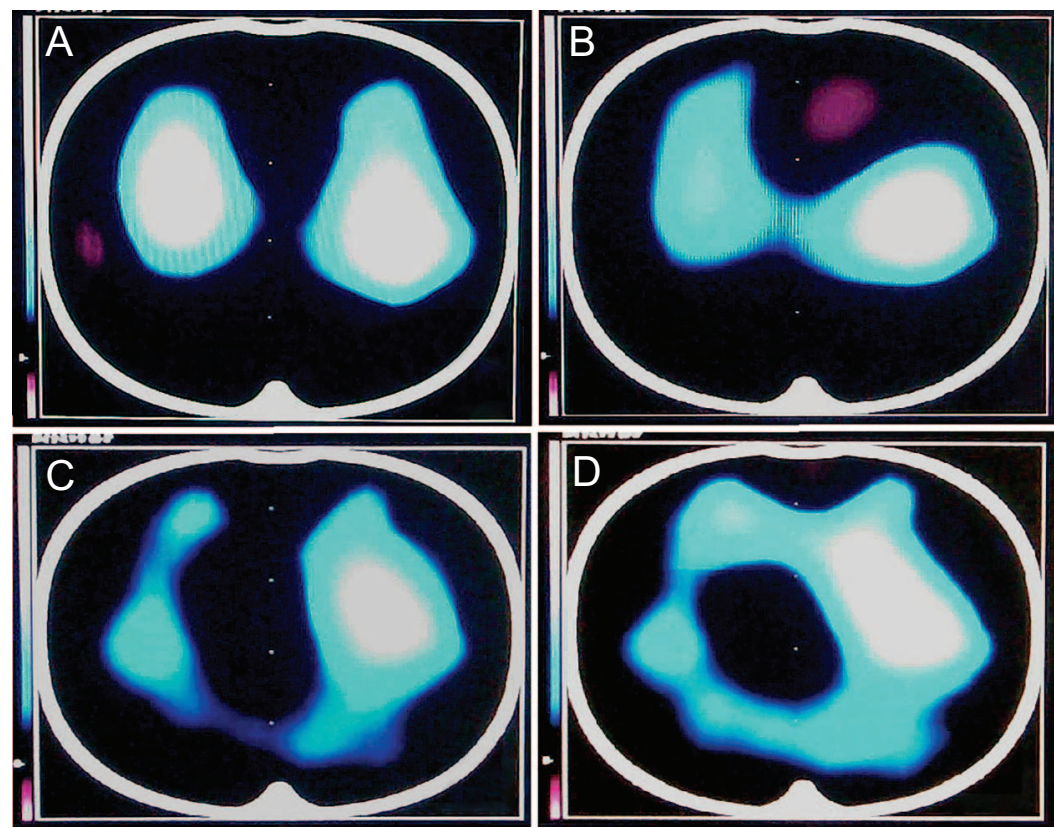

Fig. 5. Images of electrical impedance tomography for each $\mathrm{O}_{2}$ concentration. A: $5 \mathrm{~L} / \mathrm{min}$ (standard $\mathrm{O}_{2}$ ), $\mathrm{B}: 30 \mathrm{~L} / \mathrm{min}, \mathrm{C}: 40 \mathrm{~L} / \mathrm{min}$, and D: $50 \mathrm{~L} / \mathrm{min}$ (HFNC therapy). HFNC = high-flow nasal cannula.

and started walking on postoperative day 1 . The loss of lung volume was reduced by walking because of the effect of gravity on the dorsal region of the lung and para-diaphragm. Early extubation is known to improve hemodynamics, especially in patients undergoing coronary artery bypass graft and its safety has now been reported. ${ }^{23}$ The authors also believe that early extubation is safe if the patient's status is stable (ie, no active bleeding, adequate mechanical ventilation using CPAP, and a normal level of consciousness). Invasive ventilation has an adverse effect on hemodynamics (eg, by reducing blood pressure); therefore, early extubation with HFNC support improves hemodynamics. We hypothesized that early postoperative recovery and improved postoperative oxygenation and atelectasis would be associated with the effects of HFNC therapy. In this study, the length of hospital stay was shorter for HFNC subjects than for the subjects receiving standard $\mathrm{O}_{2}$, but this difference was not significant $(P=.056)$. The lack of differences in postoperative hospitalization between these 2 groups might be due to our implementation of ERAS management for both groups.

Moreover, the number of subjects requiring postoperative diuretic therapy was significantly lower in the HFNC group indicating that HFNC therapy can reduce the amount of pleural fluid. However, this finding might be the result of early rehabilitation, shortening of the duration of oxygen therapy, and the increase in insensitive sweating.

Our institution has adopted the target oxygen saturation method described by the British Thoracic Society. ${ }^{24}$ However, it is not necessary to maintain $\mathrm{S}_{\mathrm{pO}_{2}}$ close to $100 \%$. As mentioned earlier, removal of the cannula is more useful in terms of decreasing the stress to the subject and increasing their motivation to start rehabilitation.

A systematic review and meta-analysis of randomized controlled trials that evaluated the effect of HFNC therapy on the need for re-intubation in adult subjects showed that HFNC therapy does not delay re-intubation and that the oxygenation level is the same as that provided by noninvasive ventilation. ${ }^{25}$ None of the subjects in our study had respiratory failure; therefore, the lung status of subjects in this study might be better than that of subjects in respiratory failure. Hence, for patients with respiratory failure, the indication for HFNC therapy should considered more carefully.

This study has some limitations. All subjects in the study had undergone off-pump coronary artery bypass graft surgery, so the effect of HFNC therapy in those who undergo on-pump cardiac surgery could not be evaluated. Furthermore, patients with chronic kidney disease without dialysis (ie, serum creatinine $\geq 2.0 \mathrm{mg} / \mathrm{dL}$ ) were excluded. These patients may develop more pleural effusion after surgery, which could increase the atelectasis volume. However, we believe that HFNC therapy would also reduce pleural effusion and atelectasis in these patients.

\section{Conclusions}

HFNC therapy with early extubation in subjects who have undergone coronary artery bypass graft surgery could improve postoperative atelectasis, shorten the duration of 


\section{HFNC in Subjects Post Off-Pump CABG}

oxygen therapy, and reduce the total amount of oxygen administered compared to standard oxygen inhalation therapy. HFNC therapy might lead to an earlier return to activities of daily living and may decrease the postoperative hospital stay. However, this study does not provide definitive proof, so further research is necessary.

\section{REFERENCES}

1. Kendall F, Oliveira J, Peleteiro B, Pinho P, Bastos PT. Inspiratory muscle training is effective to reduce postoperative pulmonary complications and length of hospital stay: a systematic review and metaanalysis. Disabil Rehabil 2018;40(8):864-882.

2. Maggiore SM, Idone FA, Vaschetto R, Festa R, Cataldo A, Antonicelli $F$, et al. Nasal high-flow versus Venturi mask oxygen therapy after extubation. Effects on oxygenation, comfort, and clinical outcome. Am J Respir Crit Care Med 2014;190(3):282-288.

3. Parke R, McGuinness S, Eccleston M. Nasal high-flow therapy delivers low level positive airway pressure. Br J Anaesth 2009;103(6): 886-890.

4. Ni YN, Luo J, Yu H, Liu D, Ni Z, Cheng J, et al. Can high-flow nasal cannula reduce the rate of endotracheal intubation in adult patients with acute respiratory failure compared with conventional oxygen therapy and noninvasive positive pressure ventilation? A systematic review and meta-analysis. Chest 2017;151(4):764-775.

5. Parke RL, Eccleston ML, McGuinness SP. The effects of flow on airway pressure during nasal high-flow oxygen therapy. Respir Care 2011;56(8):1151-1155.

6. Ritchie JE, Williams AB, Gerard C, Hockey H. Evaluation of a humidified nasal high-flow oxygen system, using oxygraphy, capnography and measurement of upper airway pressures. Anaesth Intensive Care 2011;39(6):1103-1110.

7. Groves N, Tobin A. High-flow nasal oxygen generates positive airway pressure in adult volunteers. Aust Crit Care 2007;20(4):126131.

8. Spence CJT, Buchmann NA, Jermy MC, Moore SM. Stereoscopic PIV measurements of flow in the nasal cavity with high flow therapy. Exp Fluids 2011;50(4):1005-1017.

9. Schwabbauer N, Berg B, Blumenstock G, Haap M, Hetzel J, Riessen R. Nasal high-flow oxygen therapy in patients with hypoxic respiratory failure: effect on functional and subjective respiratory parameters compared to conventional oxygen therapy and non-invasive ventilation (NIV). BMC Anesthesiol 2014;14:66.

10. Dysart K, Miller TL, Wolfson MR, Shaffer TH. Research in high flow therapy: mechanisms of action. Respir Med 2009;103(10):14001405.

11. Hernández G, Vaquero C, Colinas L, Cuena R, González P, Canabal A, et al. Effect of postextubation high-flow nasal cannula vs. noninvasive ventilation on reintubation and postextubation respiratory failure in high-risk patients: a randomized clinical trial. JAMA 2016; 316(15):1565-1574.

12. Goepfert MS, Reuter DA, Akyol D, Lamm P, Kilger E, Goetz AE. Goal-directed fluid management reduces vasopressor and catechol- amine use in cardiac surgery patients. Intensive Care Med 2007; 33(1):96-103.

13. Kataoka G, Murai N, Kodera K, Sasaki A, Asano R, Ikeda M, et al. Clinical experience with Smart Care after off-pump coronary artery bypass for early extubation. J Artif Organs 2007;10(4):218-222.

14. Corley A, Caruana LR, Barnett AG, Tronstad O, Fraser JF. Oxygen delivery through high-flow nasal cannulae increase end-expiratory lung volume and reduce respiratory rate in post-cardiac surgical patients. Br J Anaesth 2011;107(6):998-1004.

15. Zochios V, Klein AA, Jones N, Kriz T. Effect of high-flow nasal oxygen on pulmonary complications and outcomes after adult cardiothoracic surgery: a qualitative review. J Cardiothorac Vasc Anesth 2016;30(5):1379-1385.

16. Landquist H, Hedenstierna G, Strandberg A, Tokics L, Brismar B. $\mathrm{CT}$-assessment of dependent lung densities in man during general anaesthesia. Acta Radiol 1995;36(6):626-632.

17. Joshi P, Fraser JF, Mullany DV. The high risk cardiac surgical patient. Curr Anaesth Crit Care 2005;16(6):369-383.

18. Futier E, Paugam-Burtz C, Godet T, Khoy-Ear L, Rozencwajg S, Delay JM, et al. Effect of early postextubation high-flow nasal cannula vs conventional oxygen therapy on hypoxaemia in patients after major abdominal surgery: a French multicentre randomised controlled trial (OPERA). Intensive Care Med 2016;42(12):1888-1898.

19. Curley GF, Laffy JG, Zhang H, Slutsky AS. Noninvasive respiratory support for acute respiratory failure-high flow nasal cannula oxygen or non-invasive ventilation? J Thorac Dis 2015;7(7):1092-1097.

20. Tatsuishi W, Kohri T, Kodera K, Asano R, Kataoka G, Kubota S, Nakano K. Usefulness of enhanced recovery after surgery protocol for perioperative management following open repair of abdominal aortic aneurysm surgery. Surg Today 2012;42(12):1195-1200.

21. Moon MC, Abdoh A, Hamilton GA, Lindsay WG, Duke PC, Pascoe EA, Del Rizzo DF. Safety and efficacy of fast track in patients undergoing coronary artery bypass surgery. J Card Surg 2001;16(4): 319-326.

22. Celkan MA, Ustunsoy H, Daglar B, Kazaz H, Kocoglu H. Readmission and mortality in patients undergoing off-pump coronary artery bypass surgery with fast-track recovery protocol. Heart Vessels 2005; 20(6):251-255.

23. Borracci RA, Ochoa G, Ingino CA, Lebus JM, Grimaldi SV, Gambetta MX. Routine operation theatre extubation after cardiac surgery in the elderly. Interact Cardiovasc Thorac Surg 2016; 22(5):627-632.

24. O'Driscoll BR, Howard LS, Earis J, Mak V, British Thoracic Society Emergency Oxygen Guideline Group; BTS Emergency Oxygen Guideline Development Group. BTS guideline for oxygen use in adults in healthcare and emergency settings. Thorax 2017;72(Suppl 1):ii1-ii90.

25. Huang HW, Sun XM, Shi ZH, Chen GQ, Chen L, Friedrich JO, Zhou JX. Effect of high-flow nasal cannula oxygen therapy versus conventional oxygen therapy and noninvasive ventilation on reintubation rate in adult patients after extubation: a systematic review and meta-analysis of randomized controlled trials. J Intensive Care Med 2018;33(11):609-623. 